\title{
Britische Spitäler und Polikliniken als Heil- und Forschungsstätten 1720-1820
}

\author{
Von Ulrich Tröhler
}

\section{I}

Die Gründung einer großen Anzahl Spitäler (general hospitals, infirmaries) und Polikliniken (dispensaries) im England des 18. Jahrhunderts ist wohlbekannt ${ }^{1}$. Buer sprach schon 1926 von einer eigentlichen «Spitalbewegung», und es ist eine Tatsache, daß allein während der Regierungszeit Georgs III. (1760-1820) in London die Zahl der Spitäler ${ }^{2}$ von 13 auf 17 und jene der Polikliniken von 0 auf mindestens 34 anstieg. Für die Provinz lauten die entsprechenden Zahlen 16 und 61 für die Spitäler und 0 und 36 für die Polikliniken, wobei zu bedenken ist, daß in beiden Fällen auch die meisten vor 1760 bestehenden Institutionen erst nach 1720 gegründet worden waren ${ }^{3}$. Unter diesen Neugründungen befanden sich nicht ausschließlich Allgemeinspitäler, sondern auch spezialisierte Institutionen. Die Entwicklung der letzteren geht aus der Tabelle hervor, aus der sich auch die zahlenmäßig erheblichsten Probleme der Zivilmedizin jener Zeit herauslesen lassen.

Tabelle: Spezialisierte Spitäler und Polikliniken in Großbritannien bis 1820 *

Gebiet

Rheumatische Krankheiten

Geburtshilfe

Pocken

Geschlechtskrankheiten

Kinderkrankheiten

«Fieber»

Tuberkulose

Krebs

Augenkrankheiten

Augen- und Ohrenleiden

Ohrenleiden
Mindestanzahl

Erstgründung

1738

1745

1746

1746

1769

1783

1791

1801

1771

1804

1816

* ohne Spezialabteilungen an Allgemeinspitälern 
Diese Spitalbewegung ist in mehr als einer Hinsicht eines der hervorstechendsten Merkmale der damaligen britischen Medizin. Wegen der im Zusammenhang mit dem Bruch mit der katholischen Kirche im 15. Jahrhundert erfolgten Auflösung von Spitälern mittelalterlicher Ausrichtung bestand in England ein gewisser Nachholbedarf. Wesentlich aber ist, daß diese Gründungen einen neuen Zug im Spitalwesen anzeigen, der sich in England mehr als anderswo im 18. Jahrhundert feststellen läßt: Die Pflege von Kranken tritt anstelle der mittelalterlichen Praxis der Fürsorge für Arme und Unglückselige jeder Gattung in den Vordergrund ${ }^{4}$. Bei den Polikliniken ist dies klar der Fall. Diese «Medikalisation» wird indessen auch bei den Spitälern, namentlich aus ihrer Organisation, ersichtlich: Tendierten die traditionellen großen Allgemeinspitäler dahin, von Laien, speziell von den Donatoren, dominiert zu werden, so waren die kleinen, spezialisierten Institutionen meist auf Betreiben einzelner Ärzte zustande gekommen und wurden nachher auch von ihnen beherrscht ${ }^{5}$.

II

Es ist noch offen, inwiefern diese Gründungen allein mit der Reihe sozioökonomischer Veränderungen zusammenhingen, die man gemeinhin unter dem Begriff der frühen industriellen Revolution zusammenfaßt und welche die noch heute andauernde Verstädterung der Bevölkerung in Gang brachten $^{6}$. Jedenfalls läßt sich nicht von der Hand weisen, daß die Leiden der armen Bevölkerung, einst in ländlicher Einsamkeit verloren, nun in der Masse augenfällig wurden. Aber die Veränderungen, die zu diesem Notstand führten, sorgten in gewisser Hinsicht auch für dessen Bekämpfung durch humanitäre Reformen, die ihrerseits den Fortschritt in medizinischer klinischer Wissenschaft erst ermöglichten.

Dazu hatte bislang unter anderem das Instrument gefehlt, das erlaubte, klinische, pathologische und therapeutische Daten über eine Krankheit in weniger als einem Lebensalter zusammenzutragen. Dies hing von Spitälern mit genügend Beobachtungsmöglichkeiten ab, die wiederum auf das Wachsen der Städte warten mußten ${ }^{7}$.

Die Stadtspitäler waren indessen keine ausschließliche Bedingung für den Fortschritt. Die Militärmedizin und die Privatpraxis in Städten boten die gleichen Voraussetzungen. Das zeigt, daß neben den materiellen Bedingungen auch der Wille zur eigenen Beobachtung und der Glaube daran ausschlaggebend waren. So ist die Rolle der Spitäler in der unbestrittenen Bevölkerungszunahme während der zweiten Jahrhunderthälfte noch Ge- 
genstand von Diskussionen. Hinweisen für eine positive Wirkung auf die Bevölkerungszunahme steht die Auffassung gegenüber, die Ärzte - und vorab die Spitäler hätten mehr zur Verbreitung der Krankheiten beigetragen als zu ihrer Eindämmung, geschweige denn zu ihrer Heilung ${ }^{8,9}$. Ohne auf die Frage der demographischen Wirksamkeit der medizinischen Wissenschaft einzugehen, soll hier ein anderes medizinhistorisch relevantes Problem aufgegriffen werden: Äußerte sich die «Medikalisation» des Spitalwesens auch darin, daß damals bereits eine Medizin betrieben wurde, die man später, namentlich seit der Zeit der Blüte der Pariser Spitäler nach der großen Revolution, als «Spitalmedizin» bezeichnet hat? Erforschte man also in englischen Spitälern des 18. Jahrhunderts gezielt, systematisch und extensiv Krankheitsphänomene und Behandlungsmethoden?

\section{III}

Neben den angeführten institutionellen Gegebenheiten gibt es eine Anzahl weiterer Gründe, die a priori für diese Möglichkeit sprechen. Im Land der modernen Erfahrungsphilosophie wurde nun auch in der Medizin methodologisch der eigenen Beobachtung der Primat über unverifizierte Hypothesen und die Spekulation beigemessen ${ }^{10}$. Diese philosophische Grundhaltung, schon um 1830 - kontradiktorisch - als «rationeller Empirismus» bezeichnet ${ }^{11}$, findet sich ausgesprochen in den Arbeiten der führenden Lehrer der Edinburger Medizinschule seit 1720, etwa der beiden Alexander Monro (primus und secundus), William Cullens (1710-1790) und John Gregorys (1724-1773) ${ }^{12}$. Auch das langsame Aufkommen des klinischen Unterrichts, spezifisch medizinischer Fachgesellschaften und periodischer Publikationen ging von Edinburgh aus ${ }^{13}$. Dem seit Ende des 17. Jahrhunderts von Laienseite in der Royal Society angefachten Interesse an Demographie und Sterblichkeitsstatistik gaben sozialmedizinische Bemühungen der Aufklärungszeit, wie die Inokulation der Pocken in England seit 1722, Auftrieb unter den Ärzten ${ }^{14}$.

Hinwendung zu den Dingen dieser Welt zeichnete ja die Aufklärungszeit allgemein aus. In England waren eine Anzahl sozialer und administrativer Reformbestrebungen im Gange. Im Lichte der Zunahme des Handels, der Zivilschiffahrt, der Entwicklung des Versicherungswesens und des damit verbundenen Fortschrittsglaubens entsprachen sie einem Zeitgeist, der als «allgemeiner Wunsch nach Effizienz» bezeichnet worden ist ${ }^{15}$.

Dieser Geist widerspiegelt sich auch im Spitalwesen. Die englischen Spitäler waren wenigstens teilweise das Resultat der Philanthropie einer 
begüterten Mittel- und Oberschicht. Aber diese Philanthropie änderte nun ihren Charakter. Die paternalistische Almosengabe wandelte sich zur modernen, kalten Philanthropie mit strikter Organisation und genauen Zielen. Wie ein Historiker es ausdrückte, war sie nicht länger Selbstzweck, sondern mußte nun Resultate zeigen ${ }^{16}$. Neben philanthropischen trugen die neuen Typen medizinischer Einrichtungen, Polikliniken und Spezialspitäler auch sozialreformerische Züge. Wäre es auch deswegen nicht naheliegend, wenn man dort auf Resultate-und, weshalb nicht, auf Forschung-Gewicht legte? Der Gedanke leuchtet um so mehr ein, wenn man an die Ärzte dieser Einrichtungen denkt, die aufgeklärtesten ihrer Zeit, die meist in Edinburgh studiert hatten ${ }^{17}$.

Von allen diesen Gegebenheiten ausgehend, soll in der vorliegenden Arbeit der Frage nach «Spitalmedizin» vorab am Beispiel der «Fieber» nachgegangen werden, denn sie bildeten damals die quantitativ wichtigsten medizinischen Probleme. Das Ergebnis soll so dargestellt werden, wie es für die Zeitgenossen selbst ersichtlich wurde, nämlich aus veröffentlichten Arbeiten einer Auswahl der an jenen neuen Institutionen tätigen Ärzte.

\section{IV}

Als erste allgemeine Poliklinik ${ }^{18}$ in England wurde 1770 das General Dispensary in Aldersgate Street am Rande der Londoner City eröffnet. Als Ärzte figurierten John Coakeley Lettsom (1744-1815) und James Sims (1741-1820 ${ }^{19}$. Lettsom, ein Quäker, ist mehr als Organisator und als Sozialreformer wichtig denn als eigentlicher Wissenschaftler ${ }^{20}$. «On the improvement of medicine in London on the basis of public good» lautete der Titel eines 1775 von ihm verfaßten Pamphlets. In der Tat betrachtete Lettsom seine Poliklinikgründung als geeignetes Mittel zur Erreichung dieses Zieles, und seine Schrift enthielt vier Hauptbegründungen. Erstens würde durch eine solche Institution die Sterblichkeit im betreffenden Stadtteil gesenkt (was er zahlenmäßig bewies). Zweitens geböten diese Erfolge der Quacksalberei Einhalt. Drittens könnten Polikliniken zum klinischen Unterricht herangezogen werden, woran es in London sehr gebreche. (Lettsom fügte denn auch einen detaillierten Organisationsplan bei, der unter anderem das Führen genauer klinischer Register durch die Studenten vorsah.) Und schließlich, bemerkte er, gestatteten solche Institute, den Wert von Theorien in großangelegten Experimenten zu prüfen, woraus sich die Wirksamkeit oder Unwirksamkeit gewisser Heilmittel für bestimmte Krankheiten mit Sicherheit ergäbe ${ }^{21}$. Durch klinische Ausbil- 
dung und Therapieforschung versprach er sich also allgemein einen meßbaren Erfolg ${ }^{22}$.

Ins gleiche Horn stieß John Millar (1733-1805), der Arzt des Westminster General Dispensary, der nächsten in London eröffneten Poliklinik (1774): «Obschon die Behandlung der einzelnen der ausdrückliche und unmittelbare Zweck dieser Institution war», schrieb er, «beabsichtigten wir doch, wichtigere und weitergehende Ziele zu erreichen», nämlich ein besseres Verständnis der hauptsächlichen Krankheiten und den Nachweis sicherer und wirkungsvollerer Behandlungsmethoden ${ }^{23}$. Zusammen mit William Black (1749-1829), der zeitweise an einer Geburtshilfeanstalt tätig war, hielt Millar die Analyse der «gesammelten Krankengeschichten von Spitälern, Polikliniken und Einzelpraxen» mit Hilfe einfacher Arithmetik für den sicheren «Führer und Kompaß durch das Labyrinth der Therapie». ${ }^{24}$ Dieser Meinung war auch William Rowley (1742-1806) vom St. Marylebone Dispensary in London (1784) ${ }^{25}$. George Fordyce (1736-1802) beschrieb 1793 ein tabellarisches System zur Ordnung klinischer Beobachtungen und Erleichterung ihrer Auswertung in seinem «attempt to improve the evidence in medicine». ${ }^{26}$

Ähnliche Stimmen ließen sich auch aus der Provinz vernehmen. In Newcastle verfaßte der Gründer einer Poliklinik (1777), John Clark (1744-1805) ${ }^{27}$, einen programmatischen Appendix über «the method of improving medical returns» zu seinen «Observations on fevers ...» $(1780)^{28}$. In Chester veröffentlichte 1784 John Haygarth (1740-1827), der Verantwortliche der «Fieberabteilung» am Spital, einen Plan zur summarischen Aufzeichnung der behandelten Fälle, den er seit 1767 erprobt hatte ${ }^{28 a}$. In Manchester sprach sich 1792 John Ferriar (1761-1815) ${ }^{29}$, der Mitbegründer des dortigen Fieberspitals und spätere Arzt an der Infirmary, gegen den Verlaß auf Einzelfälle aus, wo doch öffentliche Spitäler «die günstigste Gelegenheit bieten, mit Präzision viele Einzelheiten in der Krankheitsbeschreibung festzustellen und den Wert überlieferter Therapieformen zu überprüfen ..., indem man eine beträchtliche Anzahl Patienten ... studiert». Dies hatte mit Zahlen zu geschẹhen, denn das Gedächtnis genügte nicht ${ }^{30}$. In Stafford wollte 1795 Thomas Fowler (1736-1801) seine Spitalpraxis in den Dienst der Verbesserung der Medizin stellen ${ }^{31}$, und wiederum in Manchester empfahl Thomas Percival ${ }^{32}$ (1740-1804) von der dortigen Infirmary in seinen «Medical ethics» (1791/1803) die Analyse genauer Register (wie er sie von seinem Kollegen Ferriar kannte) für die vergleichende Bewertung des therapeutischen Erfolgs ${ }^{33}$. Gleicherweise rechtfer- 
tigte dort 1807 Samuel Argent Bardsley (1764-1850) seine numerischen «Medical Reports of cases and experiments chiefly derived from hospital practice ${ }^{34}$ und in Nottingham James Clarke $(\dagger 1818)$ seine zwischen 1807 und 1812 regelmäßig vom «Edinburgh medical and surgical Journal» abgedruckten statistischen Spitalberichte ${ }^{35}$. Aus Kreisen der Militärmedizin tönte es gleich ${ }^{36}$.

Doch auch in der Medizin sind Taten mehr als Worte. Was folgte dieser programmatischen Rhetorik, die zugegebenermaßen neohippokratischer Zuwendung zur Beobachtung, dem Baconschen Plan zur Verbesserung der Wissenschaften und der moderneren englischen Erfahrungsphilosophie entsprang ${ }^{37}$ ? Früher, schrieb Lettsom, hätte ihn «beim Verlust mit der üblichen Routine behandelter Patienten» stets das unangenehme Gefühl geplagt, daß er sie vielleicht mit einer andern Methode hätte retten können. Aber «mit meiner Wahl an das General Dispensary gab mir ein größeres Tätigkeitsfeld täglich die Gelegenheit, die Zweifel als berechtigt festzustellen und mit den früheren Schwierigkeiten aufzuräumen». ${ }^{38}$

In erster Linie ging es dabei um die Therapie der «Fieber», die ja damals nicht als Symptome, sondern als Krankheiten galten ${ }^{39}$. Einige Arten wie «ague» (Malaria) und «continuous fever» (Typhus) waren für einzelne damalige Praktiker bereits gut genug als ubiquitäre klinische Einheiten identifiziert ${ }^{40}$, so daß für einen Fall eine Zahl stehen konnte. Therapeutisch galt gegen die «Malaria» seit dem ausgehenden 17. Jahrhundert die Chinarinde als Spezifikum, während man sich bei Typhus noch an die traditionellen Evakuationen mittels Aderlaß, Schwitzen, Brech- und Abführmittel hielt. Wie schon zuvor James Lind (1716-1794), der Chefarzt am Marinespital Haslar bei Portsmouth ${ }^{41}$, begannen Lettsom und Sims am Wert dieser heroischen Typhuskuren zu zweifeln. Wie jener ersetzten sie sie durch hygienische Maßnahmen und - gaben neu Chinarinde, die Sydenham verboten hatte. Und auch sie lieferten nun den Beweis für die Sicherheit ihrer ketzerischen Behandlung mittels Spitalstatistiken. In seinen «Medical Memoirs of the General Dispensary» (1774) veröffentlichte Lettsom alle während eines Jahres behandelten Fälle in drei Tabellen. Daraus ging hervor, daß er zwischen 1773 und 1774 tatsächlich nur einen geringen Teil seiner Fieberpatienten verloren hatte ${ }^{42}$ und daß die Sterblichkeit in jener Poliklinik kleiner war als in den großen Londoner Spitälern ${ }^{43}$.

Auch John Millar unterstützte mit seiner Statistik vom Westminster Dispensary diese «milde» Therapie. Er unternahm einen wahren Kreuzzug gegen den auf bloßer Autorität beruhenden Aderlaß, wobei er insbesondere 
die sture Routine der Militärärzte aufs Korn nahm. So verglich er seine Resultate zahlenmäßig mit denjenigen englischer Militärspitäler während des Österreichischen Erbfolgekrieges (1740-1748) und des Siebenjährigen Krieges (1756-1763), die er sich im Kriegsministerium zu beschaffen wußte ${ }^{44}$. Wenig später «bewies» eine Tabelle, in der der Erfolg verschiedener Typhusbehandlungen untereinander und gegenüber bewußt unbehandelten Fällen zahlenmäßig dargestellt war, die Überlegenheit seiner Methode endgültig. Millar betonte bei dieser Gelegenheit die Notwendigkeit einer unbehandelten Kontrollgruppe. Dazu griff er auf die in den hippokratischen Schriften beschriebenen 31 der Natur überlassenen Fieberfälle zurück (wovon 15 gestorben waren). Konnte er, «for the sake of experiment», nicht auf eine Behandlung verzichten, so war er doch bereits in der Lage, entsprechende Versuche von Linds Schüler Robert Robertson (1742-1829) zu zitieren ${ }^{45}$. Auf ähnliche Weise analysierte auch Rowley sein Register an der Marylebone Infirmary ${ }^{45 a}$. Die Darstellung der Resultate Lettsoms und Millars entsprach ganz den Ideen William Blacks ${ }^{46}$. Bevor dieser sich wissenschaftlich der klinischen Medizin und der Demographie zuwandte, hatte er über den Wert der Pockeninokulation gearbeitet ${ }^{47}$, ein während des ganzen 18. Jahrhunderts statistisch behandeltes Gebiet. Er gab an, daß die Polemiken darüber sein Interesse für die Anwendung arithmetischer Analysen auf andere medizinische Fragen geweckt hätten ${ }^{48}$. Seine 1788 gehaltene Festansprache «Comparative view of the mortality of the human species at all ages» arbeitete er im gleichen Jahr zu einer «Arithmetical and medical analysis of the diseases and mortality of the human species ...» aus, einem Buch, das zum größeren Teil klinischen und nur zum kleineren Teil demographischen Inhalts war. Beide Teile bauten auf zahlenmäßiger Analyse auf. Die klinischen Daten hatte Black dort gefunden, wo es sie gab: Für die bessere Beschreibung und Abgrenzung ${ }^{49}$ der Fieber, des Asthmas, der Wassersucht und der Gelbsucht dienten ihm die Register von Lettsoms Poliklinik, für Keuchhusten jene des Armstrongschen Dispensary für Kinder. Für «Palsy» benutzte er die Daten von Rice Charleton (1710-1789), erhoben am National Hospital for Rheumatic Diseases in Bath (siehe Tabelle), für Nierenleiden diejenigen Matthew Dobsons (1735-1785) von der Norwich and Norfolk Infirmary. Was schließlich die Geisteskrankheiten betrifft, machte er sich selbst an die Auswertung der Register des Bethlehem Asylum («Bedlam») in der Nähe Londons ${ }^{50}$. Nach über 400 Seiten bereute Black, die Chirurgie nicht gleich behandeln zu können ... «Auf Abszeß und Ulcera ..., von denen es eine solche Unzahl in unseren Spitälern gibt, auf 
Krebs und Lithotomie wollte ich den sicheren und aussagekräftigen Test der Medizinischen Arithmetik durch und durch anwenden», schrieb er $1789^{51}$.

Blacks Schlagwort von der «Medizinischen Arithmetik» war offensichtlich an Sir William Pettys «Politische Arithmetik» angelehnt. Dies ist kaum ein Zufall, denn sowohl er wie Millar schrieben wiederholt im gleichen Stil über politische und militärische (Reform)themen während des Revolutionskrieges der 1790er Jahre, was die Verwandtschaft der medizinischen mit der politischen Arithmetik unterstreicht ${ }^{52}$.

Blacks Überzeugung von der entscheidenden Rolle des arithmetischen Beweises zeigt sich in der Änderung seiner Typhustherapie. Hatte er noch 1782 den Aderlaß empfohlen, so warnte er 1789, daß in dieser Beziehung «sogar jenem ausgezeichneten modernen Autor, Pringle ${ }^{53}$, wie Dr. Millar beweist, mit äußerster Vorsicht zu folgen ist». ${ }^{54}$

Millar und Black kannten sich, und Millar seinerseits zitierte Blacks bemerkenswerte Metapher: «Wenn es eine mathematisch fundierte Begründung gibt, ist es eine ebenso große Verrücktheit, eine andere zu gebrauchen, wie im Dunkeln herumzutappen, wenn man eine Kerze zur Hand hat.» ${ }^{55}$

Blacks Buch kann füglich als das Lehrbuch einer arithmetisch (d.h. statistisch) ${ }^{56}$ begründeten klinischen Medizin bezeichnet werden. Da alle Protagonisten dieser Auffassung den Primat der beobachteten Tatsachen über spekulative Theorien betonten, liegt es nahe, sie im Sprachgebrauch des 18. Jahrhunderts «arithmetische Observationisten» zu nennen ${ }^{57}$. Dies um so mehr, als sie mit einer eigenen Gesellschaft auch formal ihre Absichten bekundeten: Es war dies die heute noch bestehende Medical Society of London ${ }^{58}$, gegründet 1773 von Lettsom, deren sukzessive Präsidenten Millar, Lettsom und Sims waren und in der Black 1788 die obenerwähnte Festansprache hielt. Eine ähnliche, aber lockerere Verbindung bestand unter den in der Provinz gleiche Ziele verfolgenden Spitalpraktikern: Sowohl Ärzte wie auch Chirurgen trafen sich regelmäßig im günstig gelegenen Städtchen Warrington ${ }^{59}$.

Unter den Ärzten waren es die Gründer von Fieberspitälern: Clark, James Currie (1756-1805), Ferriar, Haygarth und Percival. Solche Spitäler gab es in der Provinz, gab es in Newcastle, Liverpool, Manchester und Chester schon vor der Gründung des «London Fever Hospital» von 1802; dieses entstand unter dem Einfluß von Clark, Currie und Lettsom. Damit sind auch die Querverbindungen zwischen den beiden Gruppen angedeutet. Die Mitglieder standen untereinander in brieflichem und persönlichem 
Kontakt, und die Provinzleute hielten Vorträge und publizierten in den Memoirs der Medical Society ${ }^{60}$.

Die Fieber bildeten ein wesentliches Feld für diese frühe spitalorientierte systematische Forschung, aber nicht das einzige. Thomas Fowler (1736-1801) berichtete zwischen 1785 und 1795 in drei Medical Reports über die Wirkung des Tabaks, Arsens sowie der Evakuationskuren bei genau bestimmten Krankheiten nach seinem klaren Programm, das Haupt- und Nebenwirkungen unterschied. Er hatte in dieser Zeit 16000 verschiedene Fälle in der Stafford Infirmary registriert, die er nun systematisch auswertete. So führte er die heute nach ihm benannte Arsentinktur anhand von 247 in einer Tabelle analysierten Fällen als Ersatz für die Chinarinde in die Fiebertherapie ein. William Withering (1741-1799) war ein Vorgänger Fowlers in Stafford. Seine klassische Beschreibung der Digitaliswirkung bei «dropsy» (1785) fußte dagegen auf 163 vorweg aus der Privatpraxis stammenden Fällen. Sie waren nicht numerisch analysiert. John Ferriar aber brachte 1792 seine diesbezüglichen vergleichenden Erfahrungen an der Manchester Infirmary zahlenmäßig in Tabellenform heraus. William Falconer (1744-1824), Charletons Nachfolger in Bath, ging sogar so weit, die Wahrscheinlichkeit und den Grad des Erfolgs einer Badekur bei rheumatischen Leiden - und die Gefahr eines zweiten Insults bei Apoplektikern anhand der Spitalregister nach Jahreszeit, Alter und Geschlecht des Patienten auf vier Kommastellen genau auszurechnen. Und John Haygarth stellte in Bath einen Placebo-Versuch betreffend die modischen «metallic tractors» des Dr. Perkin, mesmerischen Andenkens, an ${ }^{61}$.

$\mathrm{V}$

Neben dieser systematisch therapeutisch-klinischen Forschung betrieben diese Autoren auch numerische Krankheitsbeschreibung. Black behauptete 1789 mit Recht, daß seine Tabellen aus den Bedlam Registern die einzigen numerischen und sicheren Daten seien, «die je in irgendeinem Land veröffentlicht worden, mit denen die Wahrscheinlichkeiten von Heilung, Tod und Rückfall aller Stadien jeglicher Geisteskrankheit und in jedem Alter ausgerechnet sind». ${ }^{62}$ Fowler grenzte den chronischen vom akuten Rheumatismus ab anhand des proportionellen Vorkommens der Symptome sowie der Alters- und Geschlechtsverteilung in 488 Fällen. Dabei bemerkte er auch, wie oft beginnende Fälle in chronische übergingen ${ }^{63}$. Haygarth hatte seit 176710549 Krankengeschichten tabellarisch gesammelt. 1805 beschrieb er in einer ersten seiner geplanten Auswertungen anhand von 170 
Fällen in einer Tabelle von 27 Kolonnen den akuten Rheumatismus. In einer zweiten «Clinical History» differenzierte er das Krankheitsbild der Arthritis deformans heraus. Auch wandte er die méthode numérique auf die Frage an, ob Skrofeln und Phthisis der gleichen Krankheit entsprechen. Aufgrund von 898 Fällen kam er zum Schluß, daß dem nicht so sei ${ }^{64}$.

Auf die gleiche Weise beschrieb Falconer 1789 «palsy» und 1805 den «Ischias» anhand von 100 beziehungsweise 556 Fällen ${ }^{65}$. Dobson benützte das Register seines Spitals zur Erforschung der Ursachen des Blasensteins ${ }^{65}$. Sir John Baker (1722-1804) erkannte dank seinen Aufzeichnungen an der Devon und Exeter Infirmary zwischen 1762 und 1767 den Zusammenhang zwischen der sogenannten Devonshire-Kolik und den Bleifassungen der Mostfässer. Der Erfolg der Präventivmaßnahmen zeigte sich wiederum in den offiziellen Spitalstatistiken aus Exeter und Bath ${ }^{66}$. Dobsons chirurgischer Kollege Edward Rigby (1747-1821) unterschied mit numerischer Analyse seiner Fälle das Krankheitsbild der placenta praevia von der akzidentellen Blutung unter der Geburt. Überhaupt bemühte sich eine Reihe britischer Geburtshelfer, Fragen der Krankheitsbeschreibung, der Indikation und der Resultate therapeutischen Eingreifens auf diese Weise einer sicheren Lösung zuzuführen ${ }^{67}$.

Wie bei der Therapie nahmen auch bei der numerischen Krankheitsforschung die Fieber einen wichtigen Platz ein. Blacks Bemühungen haben wir schon erwähnt. Haygarth faßte seine Fieberabteilung in Chester (1783) als großangelegtes Experiment auf. Hatte er nicht schon 1784 die infektiöse Natur des Typhus «nach der Doktrin der Wahrscheinlichkeit» zahlenmäßig nachgewiesen ${ }^{68}$ ? Nun galt es, den Erfolg der Absonderung mittelst Mortalitätsangaben zu vergleichen. Das Londoner Fieberspital hieß mit vollem Namen «Institution for research into the cure and prevention of infectious fever». ${ }^{69}$ Thomas Bateman (1778-1821), der Chefarzt, arbeitete mit seinen Daten und denjenigen der Fieberabteilungen Alexander Marcets (1770-1822) und John Yellolys (1774-1842) am Guy's Hospital und am London Hospital. Er versuchte, objektive diagnostische Kriterien zur Unterscheidung einfacher und komplizierter Typhusformen herauszuschälen. So gab er 1818 für beide Formen in absoluten Zahlen (von 678 studierten Fällen) den Puls pro Minute, die Körpertemperatur in Fahrenheit und die vorkommenden Hauptsymptome wie Erbrechen, Durchfall, Taubheit sowie seltenere Komplikationen und die klinische Dauer in Prozenten an ${ }^{70}$. Im gleichen Jahr veröffentlichte John Cheyne (1777-1836) drei Berichte mit Daten aus zwei Dubliner Fieberspitälern. Sie illustrierten tatsächlich «die 
wunderbare Gelegenheit, welche Spitäler für die Krankheitserforschung bieten», ${ }^{71}$ denn man findet da die Körpertemperatur, Atemfrequenz und Pulsrate tabellarisch dargestellt und in Bezug gesetzt zur entsprechenden Mortalitätsrate und zu Autopsiebefunden ${ }^{72}$. Solche Arbeit entsprach einem hohen Standard numerischer Forschung, die den späteren, besser bekannten Arbeiten der Dubliner Schule von Robert Adams (1791-1875) und William Stokes (1804-1878) keineswegs nachstand.

VI

Die eingangs gestellte Frage nach systematischer klinischer Forschung in britischen Spitälern der Aufklärungszeit kann also bejahend beantwortet werden. Jedenfalls trifft dies für eine Anzahl der hier geprüften neugegründeten Allgemeinspitäler, Spezialinstallationen und Polikliniken zu. Black, Currie und Ferriar, Haygarth, Lettsom und Percival sind bisher bloß zu den britischen Pionieren der Sozialmedizin und Bevölkerungsstatistik gerechnet worden ${ }^{73}$. Ihre auf Spitalarbeit fußenden Beiträge zur Begründung der Anwendung einfacher Arithmetik in Therapie und Nosologie sind kaum beachtet geblieben. Dies gilt auch für das diesbezügliche Werk John Clarks und für Millar und Rowley überhaupt. Wir gehen deshalb nicht fehl, wenn wir sie unter die ersten Vertreter einer britischen «Spitalmedizin» einordnen.

Es war aber eine noch beträchtlichere Gruppe miteinander bekannter leitender Ärzte, die vergleichende numerische Resultate für eine notwendige Voraussetzung zur Verbesserung der berufsständischen Therapie hielt. Die dazu nötigen Patienten fanden sie in der Mehrzahl relativ schnell in den neuen Institutionen, hie und da aber auch in jahrelanger Privatpraxis. Einige benützten die numerische Methode ebenfalls zur Beschreibung von Krankheitsbildern und zur Ausarbeitung differentialdiagnostischer Kriterien.

Zugegeben, Batemans Unterscheidung zweier «Fieber» ist für uns schwierig zu verstehen. Immerhin war seine Darstellung, die proportionelle Aufzählung einzelner Symptome und Befunde, erfolgreich bei anderen Krankheiten und in der Geburtshilfe ${ }^{74}$ - und sie ist es bis heute geblieben. Diese Methode bedeutete den ersten Schritt weg von unbestimmten Feststellungen, wie «manchmal» oder «oft», zu quantitativen Angaben in Prozenten. Dies gilt natürlich auch für die Publikation therapeutischer Ergebnisse. In einer kritischen Buchbesprechung des Edinburgh Medical and Surgical Journal wird 1809 hervorgehoben: «Wahrscheinlich! - ist dies die Sprache der Wissenschaft? Wir zweifeln, ob ... [der Autor] das vergleichende Experiment oft genug gemacht hat, damit er seiner Behandlungsmethode 
sicher ist.» ${ }^{75}$ Mathematisch umfaßte die Präsentation die Summenbildung (alle Fälle einer gewissen Zeit, wobei nicht nur die erfolgreichen zu berücksichtigen waren), die Berechnung arithmetischer Mittel und - im besten Fall - von Verhältnissen (zum Beispiel zwischen Erfolg und Mißerfolg). Dazu gehörte der Vergleich mit einer unbehandelten Kontrollgruppe. Wenn es nützlich war, stellte man Tabellen zusammen als die kürzest mögliche Mitteilungsform zahlreicher Daten. Wie Haygarth 1805 bemerkte, bedeutete das Niederschreiben und Abstrahieren der Krankengeschichten eine mühselige Arbeit. «Aber», so fuhr er fort, «ich habe es nicht bereut, ... [solche Tabellen] drucken zu lassen, denn sie illustrieren die Häufigkeit der Symptome und stellen den Erfolgsgrad der verschiedenen Heilmittel mit größerer Genauigkeit dar, als irgendeine andere mir bekannte Methode.. ${ }^{76}$

Die Betonung der Nützlichkeit der Spitäler und die Verteidigung der Forschungsmethode, die sich bei allen frühen Autoren findet, weist einerseits auf eine Opposition gegen sie hin ${ }^{77}$, und andererseits zeigt sie den Neuigkeitswert, den ihr diese Ärzte selbst zusprechen. Mit dem zweiten Jahrzehnt des 19. Jahrhunderts verschwinden diese Hinweise, was wiederum andeutet, da $\beta$ die statistisch fundierte Spitalmedizin keine besondere Rechtfertigung mehr verlangte. Ein Beispiel mag dies illustrieren. Der Aderlaß bei Typhus und Kindbettfieber feierte in England ${ }^{78}$ schon ein Jahrzehnt vor der Broussaisschen «Revolution» in Frankreich ${ }^{79}$ wieder Triumphe. Henry Clutterbuck löste diese Wende 1807 zwar nicht auf statistischer Grundlage aus; doch sogleich wurde der neuentdeckte Wert des Aderlasses von den Anhängern statistisch erhärtet und andererseits von den Gegnern ebenso bezweifelt, bis sich die statistischen Aussagen die Waage hielten ${ }^{80}$. Dieses Beispiel wie auch Haygarths Forschung über Skrofeln und Phthisis zeigt die Grenzen der rein klinischen, symptomorientierten Beobachtungsmedizin, selbst wenn sie sich der numerischen Methode bediente. Solche Fragen konnten nur unter Zuhilfenahme der neuen Wissenschaften der pathologischen Anatomie und der Bakteriologie gelöst werden - immerhin nicht ohne dieselbe numerische Methode ${ }^{81}$.

Das Verdienst, die numerisch-klinische Forschungsmethode geschaffen zu haben, wird im allgemeinen der Pariser Spitalmedizin um 1830 und im speziellen Pierre-Charles-Alexandre Louis (1787-1872) zugeschrieben wegen dessen Arbeiten über Tuberkulose (1825), Typhus (1829) und über den Wert des Aderlasses $(1828,1835)^{82}$, sowie der von ihm begründeten Société Médicale d'Observation. Erstaunt es nach dem oben Dargestellten, daß Haygarth 1777 schon mit der gleichen Methode - aber verschiedenem Inhalt - 
über Phthisis zu arbeiten begann? 1805 schrieb er, er hätte dieses Gebiet deshalb aufgegeben, weil er es therapeutisch zu «melancholisch» gefunden habe. Trotzdem machte er numerische Angaben über die Alters- und Geschlechtsverteilung seiner Fälle ${ }^{83}$. Wenn auch die Aussagekraft solcher Arbeiten, teils wegen der subjektiven nosographischen Kriterien des 18. Jahrhunderts ${ }^{84}$, für uns beschränkt ist, galt dies nicht ebenso für die Zeitgenossen. In ihrem Inhalt, nicht aber in ihren Zielen und ihrer Präsentation unterscheiden sich die Forschung und Organisation der arithmetischen Observationisten von derjenigen Louis' und seiner Mitarbeiter.

Somit sind die erwähnten späteren Arbeiten der Dubliner Schule wohl weniger durch Ausstrahlung der Pariser Numeristen entstanden, sondern entsprechen mehr der eigenen und älteren britischen Tradition. Die heute als «méthode numérique de Louis» bekannte Forschungsweise kann mithin auf die aufklärerischen Ideale von Empirie, Objektivität und Fortschritt zurückverfolgt werden, die in mehreren der neugegründeten britischen Spitäler und Polikliniken während der zweiten Hälfte des 18. Jahrhunderts leitend waren.

\section{Bibliographie und Anmerkungen}

1 Gute Übersichtsarbeiten sind immer noch Chaplin, A., Medicine in England during the reign of George III, London 1919; Buer, M. C., Health, wealth, and population in the early days of the industrial revolution, London 1926; für spezifische Punkte siehe: Hartston, W., Medical dispensaries in eighteenth century London, Proc. Roy. Soc. Med. 56 (1963), 753-758; Poynter, F. N.L. (ed.), The evolution of hospitals in Britain, London 1964. Vgl. Anm.9.

2 Ohne Berücksichtigung der Irrenhäuser (asylums).

3 Tröhler, U., Quantification in British medicine and surgery 1750-1830, with special reference to its introduction into therapeutics, University College, London 1978, S. 38-40.

${ }^{4}$ Shryock, R. H., Die Entwicklung der modernen Medizin ..., Stuttgart 1947, S. 36.

${ }^{5}$ Bynum, W.F., Hospital, disease and community: The London Fever Hospital, 18011850.

${ }^{6}$ Bynum, W.F. "Introduction" to: Medicine and industrialization in history, in: E. G. Forber (ed.), Human implications of scientific advance, Proc. XVth int. Congr. Hist. Sci. Edinburgh 1977, Edinburgh 1978, S. 197-202; Webster, C., The crisis of the hospitals during the industrial revolution, ibid., S. 214-223.

7 Shryock, op. cit. Anm. 4, S. 35-37.

${ }^{8}$ Eine kürzlich erschienene Übersicht über diese Frage mit entsprechender Bibliographie findet sich in: Bynum, W.F., Health, disease and medical care, in: R.Porter and G.S. Rousseau (eds.), The ferment of knowledge, Cambridge 1980.

${ }^{9}$ London, I.S.I., The origins and growth of the dispensary movement in England, Bull. Hist. Med. 55 (1981), 322-342. 
${ }^{10}$ Shryock, op. cit. Anm.4, S.57-58; Tröhler, op. cit. Anm.3, S.35-37; Bynum, op. cit. Anm. 8.

${ }^{11}$ Bostock, J., History of Medicine, in: Forbes, J. et al. (eds.), The cyclopaedia of practical medicine, Vol.1, London 1833, p. i-Lxxii. Neuere Hinweise finden sich bei Shryock, l.c., und Milt, B., Empirie und das statistisch fundierte biologisch-medizinische Denken, Gesnerus 13 (1956), 1-28.

12 Tröhler, op. cit. Anm. 3, S.97-101.

${ }^{13}$ Fox, R.M., The rise of medical societies in Britain, in: Dr. John Fothergill and his friends, London 1919, S. 137; Gray, J., History of the Royal Medical Society, Edinburgh 1952; Dukes, C.E., London medical societies in the eighteenth century, Proc. Roy. Soc. Med. 53 (1960), 699-706; Batty-Shaw, A., The oldest medical societies in Great Britain, Med. Hist. 12 (1968), 232-244. Für eine vollständigere Liste medizinischer Gesellschaften und Periodika siehe Tröhler, op. cit. Anm. 3, S.41-43.

${ }^{14}$ Pearson, K., The history of statistics in the 17th and 18th centuries against the changing background of intellectual, scientific and religious thought, London 1978, S.543-559; Rosen, G., History of public health, New York 1958, p. 176.

${ }^{15}$ Buer, op. cit. Anm. 1, S.47-62.

16 ibid., S. 45.

${ }^{17}$ Chaplin, op. cit. Anm. 1, S.22, Hartston, op. cit. Anm.1.

${ }^{18}$ Ein früheres Ambulatorium des Royal College of Physicians zwischen 1697 und 1725 sowie das Dispensary for sick children eines George Armstrong, offen zwischen 1769 und 1781, sind hier ausgeschlossen.

${ }^{19}$ Dukes, C.E., Dr. James Sims (1741-1820): a new appraisal, Med. Hist. 5 (1961), 375-383.

${ }^{20}$ Abraham, J. J., Lettsom, his life, times, friends and descendants, London 1933.

21 ibid., S.167-175; Lettsom, J.C., On the improvement of medicine in London on the basis of public good, London 1775, S.37, 41-44, 46, 49, 51.

${ }^{22}$ Polikliniken waren in der Tat ein wichtiges Element bei der Einführung des klinischen Unterrichts in England. Siehe Cope, Z., The influence of the free dispensaries upon medical education in Britain, Hist. Med. 13 (1969), 29-36.

${ }^{23}$ Millar, J., Observations on the practice in the Medical Department of the Westminster General Dispensary, London 1777, S.231-232.

${ }^{24}$ Black, W., A comparative view of the mortality of the human species at all ages, London 1788, S. 36, 38; derselbe, An arithmetical and medical analysis of the diseases and mortality of the human species, London 1789, S.i-ii, v-vii, 261.

${ }^{25}$ Rowley, W., The causes of the great number of deaths amongst adults and children, London, 2nd ed. 1793, S. 4, 18.

${ }^{26}$ Trans. Soc. Improvement Med.-Chir. Knowledge 1 (1793), 243-293.

${ }^{27}$ Bett, W. R., John Clark (1744-1805): Medical philanthropist. Med. Press 233 (1955), 473.

${ }^{28}$ Clark, J., Observations on fever, especially those of the continued type, London 1780, S. 384.

28a Med. Obs. Inq. (London) 6 (1784), 400.

${ }^{29}$ Brockbank, E.M., John Ferriar, public health work, Tristram Shanely, other essays and verses, London 1950.

${ }^{30}$ Ferriar, J., Medical histories and reflexions, Vol. 1, zitiert in einer späteren Ausgabe, London 1810, S. XVII-XXIX. 
${ }^{31}$ Fowler, T., Medical reports on the effects of blood-letting, sudorifics, and blistening in the cure of the acute and chronic rheumatism, London 1795, S. IX.

${ }^{32}$ Brockbank, E. M., A medical statistician of a century and a half ago, Manchester 1934.

${ }^{33}$ Zitiert in: Leake, C. D. (ed.), Percival's medical ethics, Huntington, N. Y. 1975, S. 76, 88.

${ }^{34}$ London 1807, S. V.

${ }^{35}$ Siehe Tröhler, op. cit. Anm. 3, S. 468.

${ }^{36}$ Diese Aspekte sind in Tröhler, op. cit. Anm.3, Kapitel 4 und 5 (S.198-345), ausführlich entwickelt. Für die Anfänge numerischer Forschung in der Royal Navy siehe auch Tröhler, U., Towards clinical investigation on a numerical basis: James Lind at Haslar Hospital 1758-1783, Proc. XXVII Int. Congr. Hist. Med. Barcelona 1980, Vol.1, S.414-419.

${ }^{37}$ Bynum, op. cit. Anm. 8.

${ }^{38}$ Lettsom, J.E., Medical memoirs of the General dispensary in London, London 1774, S. 11.

${ }^{39}$ Zwei kürzlich geschriebene Kapitel geben einen guten Überblick über die «Fieber» vom klinischen bzw. sozialmedizinischen Standpunkt aus; in: King, L. S., The medical world of the eighteenth century, Huntington, N. Y. 1971, S. 123-155, und in: Pelling, M., Cholera fever and English medicine 1825-1855, Oxford 1978, S.1-33; s. a. Wilson, L. G., Fevers and science in early nineteenth century medicine, J. Hist. Med. 33 (1978), 386-407.

${ }^{40}$ Niebyl, P.C., The English bloodletting revolution, or modern medicine before 1850, Bull. Hist. Med. 51 (1977), 464-483.

${ }^{41}$ Tröhler, op. cit. Anm. 36.

42 Genau waren es:

3 von 22 Patienten an intermittierendem Fieber,

3 von 65 Patienten an febris nervosa,

8 von 192 Patienten an febris putrida,

0 von 82 Patienten an febris remittens,

0 von 29 Patienten an febris simplex.

${ }^{43}$ Lettsom, op. cit. Anm. 38, S. 345-348.

44 Millar, op. cit. Anm. 23, S. 14-17, 20-21, 26-30, 34.

${ }^{45}$ Millar, J., Observations on the management of diseases in the Army and Navy, London 1778-1779, S. 103-105, 188-200, 226-227, 305.

45a Rowley, op. cit. Anm. 25.

${ }^{46}$ Glass, D. V., "Introduction", in: Black, op. cit. Anm.24, 1789, Neudruck Westmead, Farnborough (Hants.) 1973.

${ }^{47}$ Black, W., Observations medical and political on the small pox and inoculation ..., London 1781

${ }^{48}$ Black, op. cit. Anm. 24, 1789, S. IV, 250.

${ }^{49}$ Wie viele Gesinnungsgenossen reduzierte er die Zahl der Fieber beträchtlich: ibid., S. 43.

50 ibid., S. 101-103, 108, 117, 139, 170, 178.

51 ibid., S. 251-264-265; op. cit. Anm.24 (1788), S. 407.

52 Tröhler, op. cit. Anm. 3, S. 125.

${ }^{53}$ Gemeint ist Sir John Pringle (1707-1782), der Verfasser der Observations on the diseases in the army (1752), einer der angesehensten Londoner Ärzte und Wissenschaftler jener Zeit.

${ }^{54}$ Black, W., An historical sketch of medicine and surgery from their origin to the present time, London 1782, S.250; op.cit. Anm.24, 1789, S. 58. 
${ }^{55}$ Millar, J., Observations on the conduct of the war, in an appeal to the people of Great Britain..., London 1798, Vol.1, part 2, S. 76.

${ }_{56}$ Über die spätere Einführung des Wortes «statistics» ins Englische siehe Cullen, M. J., The statistical movement in early Victorian Britain, Hasocks (Ssx.) und New York 1975.

57 Tröhler, op. cit. Anm. 3, S.447-450; op. cit. Anm. 36.

${ }^{58}$ T. Hunt geht in seiner Geschichte, The Medical Society of London, London 1972, nicht auf diesen Aspekt ein.

59 Tröhler, op. cit. Anm. 3, S. 143-144.

60 ibid. S. 136.

61 ibid. S. 164-174, 179.

${ }^{62}$ Black, op. cit. Anm. 24, 1789, S. 130.

${ }^{63}$ Fowler, op. cit. Anm. 31, S.255-272.

${ }_{64}$ Tröhler, op. cit. Anm.3, S.176-178. Siehe auch Garrison and Morton's Medical Bibliography, No.4492.

65 Tröhler, op. cit. Anm. 3, S. 375-378.

66 ibid. S. 49.

67 Tröhler, U., Klinisch-numerische Forschung in der britischen Geburtshilfe 1750-1820. Gesnerus 38 (1981), 69-80.

${ }^{68}$ Tröhler, op. cit. Anm.3, S.137-138; Haygarth, J., An inquiry how to prevent small-pox, Chester und London 1784, S. 25-29.

${ }^{69} \mathrm{Vgl}$. Bynum, op. cit. Anm. 5.

${ }^{70}$ Bateman, T., A succint account of the contagious fever of this country, London 1818, S. 35-36, $38,40,48-51,57,64,66,71-72$.

${ }^{71}$ Dublin Hospital Reports 1 (1818), 3.

72 ibid. 2 (1818), 110-145, auch zitiert bei Tröhler, op. cit. Anm. 3, S. 148-149.

${ }^{73}$ Vgl. Buer, op. cit. Anm.1, S.122-123; Abraham, J.J., op. cit. Anm.20; Greenwood, M., Medical Statistics from Graunt to Farr, Cambridge 1948, S.63-64; Schipperges, H., Zur Einführung statistischer Methoden in Medizin und Biologie. Die Kapsel (Hauszeitschrift Scherer GmbH, Eberbach/Baden) 27, 1115-1125, 1971.

74 S. Anm. 67.

${ }^{75}$ Edinburgh Med. Surg. J. 5 (1809), 119.

${ }^{76}$ Haygarth, J., A clinical history of diseases, London 1805, S. 187.

77 Vgl. Tröhler, op. cit. Anm. 3, S. 182.

${ }^{78}$ Niebyl, op. cit. Anm. 40.

${ }^{79}$ Ackerknecht, E.H., Broussais, or a forgotten medical revolution, Bull. Hist. Med. 27 (1953), 320-343.

${ }^{80}$ Vgl. Tröhler, op. cit. Anm. 3, S.469-470; Edinburgh Med. Surg. J.9 (1813), 458-459.

${ }^{81}$ Vgl. Wilson, op. cit. Anm. 39.

82 Rosen, G. Problems in the applications of statistical analysis to questions of health 1700-1880, Bull. Hist. Med. 29 (1955), 27-45.

Shryock, R.H., The history of quantification in Medical Science, Isis 52 (1961), 215-232. Schipperges, op. cit. Anm. 73.

Seydel, M., Statistik in der Medizin. Ein Entwurf zu ihrer Geschichte (Kieler Beitr. Gesch. Med. Pharm., Vol.15), Neumünster 1976, S. 68-69, 73-74.

${ }^{83}$ Haygarth, op. cit. Anm. 76, S. 33, 35-36. 
${ }^{84}$ Vgl. Rudolph, G., Leitgedanken der Diagnostik und Semeiotik in der französischen Medizin des 18. und frühen 19. Jahrhunderts. In: Medizinische Diagnostik in Geschichte und Gegenwart. Festschrift für H. Goerke. Münchner Beitr. Gesch. Med. Nat. Wiss., Med.-Hist. Reihe 7/8, München 1978, S.269-281. Und Henne, H., Probleme um die ärztliche Diagnose als Grundlage für die Therapie zu Ende des 18. und in der ersten Hälfte des 19. Jahrhunderts. ibid., S.283-296.

\section{Summary}

In this paper hospitals and policlinics founded between 1760 and 1820 in Britain are first cursorily described in terms of number, size and speciality. Attention is then drawn to the finding that some of their surgeons and physicians - mostly Edinburgh trained - used them as a basis for teaching students and postgraduates. In addition they consciously took advantage of these new facilities for numerical nosography and evaluation of therapy, both new and traditional, with the aid of simple statistics. Their approach to "fever" ist particularly dealt with. Because of their professed aim, i. e. "the increase of certainty of medicine" by the aid of arithmetical analysis of their own observations, this group of enlightened doctors are introduced as "arithmetical observationists". Their social organization as well as the background and significance of their method for the further development of numerical research are briefly outlined.

Priv.-Doz. Dr. med. et phil. Ulrich Tröhler

Medizinhistorische Bibliothek der Universität

Klingelbergstraße 23

C.H-4031 Basel 